\section{ENLARGEMENT OF THE MEDIASTINAL GLANDS IN CHILDREN.}

\author{
By ALLAN MONCRIEFF, \\ M.D., M.R.C.P., \\ Late Medical Registrar and Pathologist, Hospital \\ for Sick Children, Gt. Ormond St., London.
}

ENLARGEMENT of the mediastinal lymphatic glands is a comparatively common occurrence in children, and tuberculosis of these glands is the commonest form of intrathoracic tuberculosis between the ages of three and ten years. Because of the almost hidden nature of this enlargement, however, it does not attract so much attention as swelling of the lymphatic glands of the neck, for example, and yet correct treatment at an early stage is equally important in both instances.

The causes of enlargement of the mediastinal glands are, in principle, the same as for lymphatic glands elsewhere in the body. General disorders of the lymphatic system, such as lymphadenoma, for example, will not be considered here, although physical signs and symptoms referable to involvement of the mediastinal glands in such cases are similar to those discussed below. Local disease of the pulmonary tissues with secondary involvement of the glands draining the affected areas is the chief cause of swelling of the lymphatic glands at the lower part of the trachea and beginning of the main bronchi in the child. The glands on the right side are more commonly enlarged than those on the left, possibly because the presence of the aorta passing round the root of the left lung leaves less potential space for glandular swelling on this side, or because local disease is commoner in the right lung than in the left. The local causes of enlargement may be classified as simple or tuberculous.

Simple enlargement of the mediastinal glands occurs with any inflammatory lesion of the lungs or bronchi, such as bronchitis, acute or chronic, bronchiectasis, pneumonia, croupous or catarrhal, chronic pulmonary inflammation (pulmonary fibrosis) and also with asthma in many cases following an attack. The mediastinal glands are particularly involved in the catarrhal conditions of the respiratory system which occur in measles and whooping-cough and, indeed, in the latter disorder, such enlargement has been alleged by some authorities to be the cause of the "whoop." The enlargement following simple inflammatory conditions of the lungs and bronchi may remain for several months after the initial pulmonary focus has completely healed and, just as with simple enlargement of the glands in the neck, may eventually become tuberculous.

Tuberculous enlargement of the mediastinal glands is usually the result of infection of the pulmonary tissues with tubercle bacilli in the child. The inhaled organisms reach the end of a small bronchus where then "primary focus" as described by Ghon, is formed. Spread of infected material novo takes place along the lymphatic vessels draining this area and the corresponding lymphatic glands at the root of the lung enlarge. These tuberculous glands may cause no symptoms, the process may be arrested, healing take place and no further disturbance ever occur. On the other hand, the tuberculous foci present in these glands may give rise to other manifestations of tuberculosis at a later date, and it is because of this potential danger that tuberculous enlargement of the mediastinal glands is so important. Early treatment will prevent later tragedies.

The signs and symptoms of enlargement of the mediastinal glands are similar, whether this enlargement be simple or tuberculous in type, but they are much more marked in tuberculous cases. This is especially true of the symptoms, which in the latter instance are those of a chronic uncomplicated tuber- $\stackrel{\mathscr{P}}{?}$ culous infection in general. Thus the $\frac{0}{+}$ affected children fail to gain weight or 
actually lose several pounds; they suffer from loss of appetite, fretfulness and lassitude, are often slightly anæmic and show a slight, irregular evening pyrexia. Longstanding cases not infrequently show an excessive growth of downy hair on the body. In non-tuberculous cases such symptoms are all slight, and the usual story is that the affected child fails to "pick up" properly after some respiratory infection. In both instances there are added certain local symptoms or signs which are largely mechanical in origin. The most important of these is the cough which is usually harsh, hacking and paroxysmal, not at all unlike whooping-cough in many instances. In some cases more severe pressure on a bronchus may make the cough metallic and stridor may even occur. In slight cases the cough is purely catarrhal in type, and in some instances may be completely absent.

The physical signs which are reliable for the diagnosis of mediastinal glandular enlargement are few in number. Enlarged veins may be found over the upper part of the chest in front or small telangiectases over the upper thoracic spinous processes behind. Percussion may reveal some dullness over the sternum in front. or more commonly in the right paravertebral region behind, between the spinal column and the inner border of the scapula. Such impairment of the percussion note is, however, a later development and usually means that the pulmonary tissue at the root of the right lung is also involved-the so-called hilus type of tuberculosis. On auscultation harsh breath sounds over the right apex are common, with the presence of coarse râles over the roots of both lungs behind (" hilus catarrh"). Eustace Smith's sign, consisting of a venous systolic hum heard just below the right sterno-clavicular joint when the head is fully extended is unreliable, as it occurs frequently in normal children. D'Espine's sign is of more value but requires practice to elucidate. It consists of an extension downwards of the normal area over which the tracheal breath sounds can be heard behind, and it may be taken that if bronchial breathing and whispering pectoriloquy can be heard below the level of the third or fourth thoracic spinous process in a child, just to either side of the mid-line, the probable explanation is that the mediastinal glands are enlarged. A similar deduction may be made if tracheal breath sounds can be heard high up in the axilla.

Radiological examination is very important in all cases of mediastinal glandular enlargement, but the interpretation of hilus shadows requires skill and restraint. The town-bred child in an antero-posterior skiagram so frequently shows "increased hilus shadows" as to make this position valueless for the diagnosis of the carly stages of enlargement of the mediastinal glands. The oblique position is of more importance, for here, in a normal child, the posterior mediastinum should light up as a clear space during inspiration and the presence of glands can easily be detected on such examination. A skiagram taken in this position will confirm the presence of abnormal shadows in the mediastinum.

The diagnosis of enlargement of the mediastinal glands is not difficult when the clinical and radiological pictures, as outlined above, are present. It should not be made, however, on either of these findings alone, but only when both sets of observations yield clefinitely positive results. More difficult is the question of whether the enlargement is simple or tuberculous. As a general rule the diagnosis of tuberculosis should not be made within six months of an acute pulmonary infection as long as the child's general condition is improving or stationary. On the other hand, if there is a possibility of tuberculous contagion in the family circle, if the child's condition is becoming worse, or if the symptorns and signs persist for longer than six months after, say, measles or whooping-cough, then 
tuberculosis of the mediastinal glands should be suspected. Since tubercle bacilli are not being excreted in the sputum in this condition, positive proof can seldom be obtained, and the use of tuberculin in the various tests is of value only in excluding tuberculosis by a persistently negative result. Since the positive diagnosis of tuberculosis is almost impossible, it is very important that it should never be lightly made in these cases, and it is on the whole better to class all children with the signs and symptoms here described as examples of "enlarged mediastinal glands," using this as a convenient diagnosis rather than branding a child with the label of tuberculosis. Most of the legislation concerning pulmonary tuberculosis is based upon the adult type, and many authorities dealing with the social aspect fail to distinguish between phthisis in the child, which is rare, and tuberculosis of the mediastinal glands, which is the usual form. To send such children to a sanatorium where open cases of tuberculosis are being treated is thoroughly unsatisfactory. It is also important not to alarm the parents of, say, a five-year-old child, with the diagnosis of "consumption," in view of what this usually means to the general public.

The course and prognosis of enlargement of the mediastinal glands depends largely upon satisfactory treatment. If this is begun early and properly carried out, complete healing of the inflammatory process, even when this is tuberculous, is to be expected. On the other hand, if the tuberculous process does not heal, it may lead to retrograde lung infection by direct spread into the pulmonary tissues, or by entering the general circulation through a vein the tuberculous infection may lead to generalized miliary tuberculosis or to some localized acute tuberculous condition such as meningitis. The breaking-down of a tuberculous focus in the mediastinal glands may further lead to its perforation into some adjoining structure such as trachea, aorta or neso- phagus, but such results are pathologica类 curiosities. In any case the presence of tubercle bacilli in the mediastinal gland@ leads to the development of an allergic: response to the protein of these organism and consequently to an allergic type of reaction in the pulmonary tissues if re $\frac{\overline{0}}{\bar{n}}$ infection takes place in later life. As recen experiments have shown, this allergic stato is not necessarily the same as a developec immunity to the tubercle bacillus.

The hest treatment of tuberculosis in the child is naturally prevention. For practicas purposes it may be taken that intrathoracio tuberculosis in the child means infectionio from some human source, and hence th $\$$ prevention of tuberculous enlargement obo the mediastinal glands is essentially a sociaf problem. Removal of healthy children from a tuberculous environment combined witle some sort of preventive inoculation wil probably be the line of approach to this problem in the future. It is particulag se important that convalescence after meas es and whooping-cough should always $\bar{\Phi}$. adequate. Several weeks of "running wild "s in the country is the ideal to be aimed an for every case.

The curative measures for enlargement of the mediastinal glands are essentially the same whether the cause be tuberculosis of not. They consist in abundance of fresh air, exercise and good food for at least three months, and preferably for six. Since it is: often difficult to persuade parents to let $\frac{3}{\pi}$. child live with the windows widely open in? all weathers, it is usually better to send the child to the country or seaside where such treatment can be carried out, but, as saic before, the ordinary sanatorium must be avoided like the plague. During the pyrexia stage rest in bed or on a couch is desirable⿳ै but otherwise the natural exercise of children playing in the open air is of great value Heliotherapy is of benefit in many cases but between the alternatives of artificiaf sunlight baths in a smoky town and ordinary fresh air in a seaside convalescent hom 
there is no question of choice. The air of the Isle of Thanet seems to be particularly suitable for these cases.

Diet must be nourishing with plenty of protein and fat, but "stuffing" an ill child may easily upset the digestive processes and the appetite must be first gradually improved by means of an ordinary nux vomica and acid mixture. Eggs, milk, butter and cream should be taken freely and more meat given than is usual in childhood. Drugs are mostly not required, but cod-liver oil is generally beneficial and simple fruit lozenges may be used if the cough is troublesome.

The hygiene of the nose and throat must be properly attended to and definitely enlarged and diseased tonsils should be removed half-way through the convalescent period.

\section{REVIE W S.}

\section{General Practice. (Some Further EXPERIENCES). By Ernest Ward, M.D.(Camb.), F.R.C.S.(Eng.). John Bale, Sons and Danielsson, Ltd., 83-9I, Great Titchfield Street, W.I. 1930. Pp. 108. Price 3s. 6d.}

In this book the author is more particularly catering for the needs of those about to enter general practice, and all such should consider themselves privileged to read the happily inspired remarks of one so eminently fitted to offer advice.

The opening chapters deal with the important questions of the types, relative advantages, and selection of practices ; these together with certain very practical hints on the management of patients cannot fail to be of timely service to the young practitioner; the fundamentals essential to good fellowship between doctors and their colleagues is discussed at some length and here, as elsewhere throughout the book, the author emphasizes his convictions by wellchosen personal experiences.

We feel sure that this book will have, as it deserves, a wide circulation.

Gleanings from General Practice. By David Tindal, M.D., F.R.F.P.S. Glas. London : Baillière, Tindall and Cox. 1929. 8vo. Pp. 207. Price 6s. net.

In this book Dr. Tindal puts forward what he describes as a few counsels on professional conduct and medical subjects gleaned from his long experience in general medical practice; his chief concern is evidently the newly-qualified practitioner, but the scope of the book is definitely more extensive, embracing as it does many matters of practical interest which cannot fail to be of value to all ranks of the profession.

The cardinal rules of professional conduct are carefully outlined and in this context the author explicitly emphasizes the cluty, so frequently ignored, of doctors to themselves that they should safeguard their own health and physical fitness to the best of their ability. An excellent chapter deals with the more important branches of medical treatment and here, as also in his remarks on cookery, sick-room administration, \&c., Dr. 'Tindal's wide practical experience is reflected in admirable fashion.

The concluding section of the book is devoted to formulæ of useful prescriptions and these we are glad to notice have been separately indexed.

The book affords pleasant reading and valuable instruction; we can confidently recommend it to all who may be engaged in general medical practice. 